\title{
FUNDAMENTOS METAFÍSICOS DE LA COSMOLOGÍA CARTESIANA
}

Laura Benítez Grobet

INSTTUUTO DE INVESTIGACIONES FLLOSÓFICAS Universidad Naciónal áutónoma de Méxuco

El caso en el que intento fundar mi discusión es el de la cosmología cartesiana, o más específicamente, su concepción mecanicista de la naturaleza y cómo esta concepción requiere de ciertos supuestos metafísicos para su expresión cabal y sistemática.

El problema particular que enfrento y que trataré de dirimir es justamente el enfoque o perspectiva con que han de ser vistos tales supuestos metafísicos para la mejor comprensión del sistema cartesiano.

De entrada uno puede preguntarse si es lícito hablar de que se intenta lidiar con los supuestos metafísicos de la cosmología para de inmediato restringirse al mecanismo cartesiano. A este respecto hay que deslindar en la cosmología cartesiana tal y como se presenta en El Mundo o tratado de la luz, entre la cosmologia como la explicación del origen del universo, que se condensa en la teoría de los vórtices — de lo cual no habremos de ocuparnos aquí-, y de la cosmología como la explicación del mundo actual, indisolublemente ligada a la física, y a la que Descartes dedica la mayor parte del Tratado.

Si para hablar del mundo nos hemos limitado a considerar los conceptos de materia y movimiento, sus leyes y supuestos, es porque Descartes mismo nos lo autoriza al aseverar:

Sepan entonces primariamente que por naturaleza no entiendo aquí alguna deidad o alguna otra clase de poder imaginario, sino que me sirvo de esta palabra para significar la materia misma en tanto que la considero con todas las cualidades que le he atribuido comprendidas juntas, y bajo la condición de que Dios continúa conservándola del mismo modo que la ha creado... y a las reglas median. te las cuales se producen los cambios las llamo reglas de la naturaleza. ${ }^{1}$

1 Descartes, R., El Mundo o Tratado de la luz. Traducción inédita de Laura Benitez de Oeuvres, Edición de Adam \& Tanery. París, 1909, cap. VII, p: 27. 
Aunque el parágrafo anterior ofrece un buen resumen de los aspectos que nos interesa considerar en Descartes hemos de pasar a su examen. En relación con el problema de la naturaleza, lo primero que hay que decir es que para Descartes la naturaleza no es tal y como nuestros sentidos nos la muestran. En efecto, de los capítulos $1^{\circ}, 3^{\circ}$ y $4^{\circ}$ de El Mundo, Descartes discute la tesis del sentido común que acepta cómodamente que las cosas son tal y como se ofrecen a los sentidos. ${ }^{2}$ Descartes reduce este mundo de la sensibilidad, de la "variedad fenoménica", a mera apariencia.

En el capítulo III del Tratado, asienta que desde la infancia hemos tenido el prejuicio de que no hay más cuerpos alrededor de nosotros que aquellos que pueden ser percibidos, y en el IV llega a proponer una tesis en la que pretende darse cuenta genéticamente del límite del conocimiento sensorial.

Mas cuando hayan considerado mínimamente qué es lo que hace que sintamos un cuerpo o que no lo sintamos, estoy seguro de que no encontrarán en ello nada increíble [se refiere a la imposibilidad del vacío], pues conocerán evidentemente que estamos tan lejos de que todas las cosas que están alrededor de nosotros puedan ser sentidas que, por el contrario, las cosas que están más ordinariamente a nuestro alrededor son las que pueden ser menos sentidas y aquellas que siempre están alrededor de nosotros no pueden ser sentidas nunca. $^{3}$

Descartes tiene la idea de que la percepción, como cualquier otro cambio, se produce mediante el choque de partículas; por ejemplo, el aire al chocar con nuestros ojos va removiendo de ellos pequeñas partes, de manera que si en un principio tuvimos la capacidad de verlo, la acción continua de la remoción del aire hace que únicamente permanezcan aquellas partes de nuestros sentidos que resisten tal acción y, por tanto, son insensibles a ella.

Al cuestionar la percepción sensible, Descartes está en condiciones de asentar que: aunque sin ser inmediata y accesible a los sentidos,

1. Lo que verdaderamente hay es materia en movimiento o reposo.

2. La materia es extensión tridimensional y divisible al infinito.

3. Los cuerpos son aparentes en tanto lo que hay son cantidad de pequeñas partes que no dejan de moverse.

Por lo que me doy cuenta con evidencia de que no es solamente en la flama donde hay cantidad de pequeñas partes que no dejan

2 Op. cit., cap. I, p. 3.

s Ibid., cap. IV, p. 15; el subrayado es mío. 
de moverse, sino que las hay también en todos los otros cuerpos, aunque sus acciones no sean tan violentas y que a causa de su pequeñez no puedan ser percibidas por ninguno de nuestros sentidos. ${ }^{4}$

4. Todo cuerpo puede ser dividido en partes cuyo número si no es infinito, al menos para propósitos de conocimiento, es ilimitado; así, hay innumerables partes en la más pequeña porción de materia, v. gr. un grano de arena.

5. Los criterios para la distinción o diversidad de cuerpos no estarían dados por las cualidades sensibles de ellos, puesto que no son en el fondo sino agregados de partes, de donde la primera consecuencia es la homogeneidad material:

Al tratar esto deseo primeramente que adviertan que todos los cuerpos, tanto duros como líquidos, están hechos de una misma materia y que es imposible concebir que compongan nunca un cuerpo más sólido o que ocupen menos espacio. ${ }^{5}$

Por otro lado, los cuerpos se distinguen unos de otros, por ejemplo los duros de los líquidos en virtud de que "... las partes de unos pueden ser separadas en conjunto mucho más fácilmente que las de los otros". ${ }^{6} \mathrm{El}$ cuerpo duro es aquel en el que "...todas sus partes se tocan sin que quede ningún espacio entre dos de ellas y ninguna está en disposición de moverse". 7

6. La necesidad de que todas las partes de la materia se toquen está directamente relacionada con el problema del vacío. Para Descartes no hay vacío de modo que el mundo puede verse como un pleno discreto y contiguo, por más paradójica que pueda resultar semejante formulación.

En buena medida la exclusión del vacío está ligada a la necesidad de distinguir entre ciencia y magia, ya que Descartes no acepta fuerzas ocultas, causas extrañas o irracionales que actúen en la naturaleza, pero también a la de distinguir entre ciencia y religión al desechar el milagro del campo de la ciencia toda vez que significa suspensión de la ley natural. El vacío es hueco ininteligible, suspensión de la legalidad que funda la absurda concepción de la acción a distancia como lo dirá más tarde Leibniz en su disputa con Clark.

Descartes propone que si hubiera vacío, éste se daría entre las partes de los cuerpos sólidos que por su forma y por su movimiento más lento

4 Ibid., cap. III, p. 7.

5 Ibid., cap. IV, p. 11.

6 Ibid., cap. III, pp. 8-9.

7 Ibid., cap. III, p. 9. 
no alcanzan a tocarse por todas partes, pero tal situación no puede darse porque entre las partes más grandes siempre hay partes más pequeñas que tienen un movimiento más veloz, de modo que la materia es conforme un pleno. A esto sus opositores argumentaron que si todo está lleno, entonces no es posible el movimiento. Descartes contestará, como veremos, con la concepción del movimiento circular y la teoría de las partes.

7. No obstante la identificación entre materia y extensión, Descartes propone que existen tres elementos, aunque no habla como la tradición de naturalezas distintas sino que la materia es básicamente extensión tridimensional, divisible en partes según todas las figuras, con tendencia a ciertos movimientos y susceptible de recibir todos los movimientos, pero también, perfectamente concebible en reposo. Los tres elementos son: el fuego, el aire y la tierra. Pero, ¿qué son ellos realmente? Descartes nos dice:

Concibo al primero, que podemos nombrar el elemento del fuego, como un licor, el más sutil y penetrante que haya en el mundo... me imagino que sus partes son mucho más pequeñas y se mueven mucho más rápidamente que las de cualquier otro cuerpo. $\mathrm{O}$ más aún, a fin de no vernos obligados a admitir algún vacío en la naturaleza, no le atribuyo partes que tengan ningún tamaño ni figura determinada, pero me doy cuenta de que el impetu de su movimiento es suficiente para hacer que se dividan en todas las formas $y$ en todos.los sentidos por el encuentro con los otros cuerpos; y de que sus partes cambian de figura en todo momento para acomodarse a todos los lugares a donde entran, de suerte que no hay jamás paso demasiado estrecho ni ángulo suficientemente pequeño entre las partes de los otros cuerpos donde los de este elemento no penetren sin ninguna dificultad y que llenen exactamente. ${ }^{8}$

El aire también es un licor sutil pero sus partes tienen un tamaño medio y figura definida (redonda). Entre las partes del aire quedan pequeños instersticios en los que se desliza el primer elemento; por tanto, es siempre menos puro pues contiene materia del primero.

En cuanto a la tierra como elemento, sus partes son mucho más grandes y estorbosas, se mueven lentamente o incluso no se mueven.

En suma, los criterios de diferenciación de los elementos son los principios explicativos de la materia concebida como este pleno de partes que se ditinguen no por naturaleza intrínseca o diferenciación cualitativa, sino por la velocidad de su movimiento, su tamaño, su figura y el modo en que sus partes se acomodan.

8 Ibid., cap. V, p. 17. 
Hay en la concepción de los elementos de Descartes una dualidad interesante: por un lado, los tres elementos no se identifican con los cuerpos que comúnmente llamamos aire, agua y tierra, pues los que percibimos sobre la tierra no son puros, sino productos de mezcla que se dan en la superficie de los cuerpos puros al entretocarse los distintos elementos. Por otra parte, los elementos como meros conceptos teóricos encarnan en los cuerpos celestes concretos; así, el primer elemento es el constitutivo del sol y las estrellas, el segundo de los cielos y el tercero de los planetas y cometas.

A partir de lo propuesto hasta el momento, especialmente la caracterización de la naturaleza como materia, nos damos cuenta de que en Descartes se trata de una concepción que se aparta del sentido común tanto como de lo meramente perceptual y busca reducir la complejidad del mundo a términos evidentes que borran la diversidad cualitativa para erigirse en explicación unitaria de la totalidad variante y variable del universo.

Cuando Descartes describe el mundo no sólo nos va a hablar de la materia sino también del movimiento y diferencia claramente entre descripción del movimiento y causa del movimiento. Así, al explicar el movimiento dice: "Todo ha comenzado a moverse tan pronto como el mundo comenzó a existir." "

A partir de los supuestos básicos en torno a la materia, tales como que no puede haber espacio vacío en vista de que espacio significa extensión y extensión es ser extenso o materia, que la extensión es divisible siempre, por lo cual no es posible concebir átomos absolutos, o en otras palabras, que es inconcebible un límite a la divisibilidad, $y$, finalmente, que de suyo la extensión dice ilimitación, se sigue la necesidad cartesiana de reducir los cambios de la naturaleza a cambios de la materia tal y como la concibe, de donde toda modificación no puede ser sino desplazamiento de las partes, e incremento y pérdida de velocidad. Descartes tendrá que explicitar las leyes del movimiento de la materia entendida como pleno discreto y contiguo.

El movimiento que Descartes va a tomar en cuenta es el movimiento local, pues todos los otros movimientos admitidos por Aristóteles y la escolástica no son en su concepto para nada evidentes.

Confiesan ellos mismos [se refiere a los filósofos ecolásticos] que la naturaleza del suyo [del movimiento que explican] es muy poco conocida, y que para hacerla en alguna forma inteligible, no la han sabido explicar aun más claramente que en estos términos: Motus est actus entis in potentia, prout in potentia est, los cuales son para

9 Ibid., cap. III, p. 7. 
mí tan oscuros que me veo obligado a dejarles aquí con su "jerga" puesto que no podría interpretarlos ( $y$, en efecto, estas palabras: el movimiento es el acto de un ser en potencia, en tanto está en pa tencia, no son más claras por estar en francés). ${ }^{10}$

El movimiento local no se define como la acción de pasar de un lugar a otro, sino más bien como cambio de posición. Dice Descartes:

Yo no conozco ningún [tipo de movimiento] que sea más fácil de concebir que el de las líneas de los geómetras que hacen que los cuerpos pasen de un lugar a otro y ocupen sucesivamente todos los espacios que están entre dos. ${ }^{11}$

En los Principios la noción se afina mucho más, ahí las nociones de disposición de unos cuerpos frente a los otros, la relatividad del movimiento y el tratamiento del movimiento como una relación, se hacen más patentes. No obstante, lo que más me interesa enfatizar es que Descartes separa, en cierta forma, el movimiento de la acción, al desechar cualquier causa interna y oculta como explicación; el movimiento se reduce “. . a la simple consideración geométrica de un cambio de posición". ${ }^{12}$

La tarea fundamental de la física cartesiana es reducir todos los efectos y propiedades de los cuerpos que conocemos por experiencia a partes de materia (cuerpos como porciones limitadas de extensión) con determinada figura, tamaño, posición con respecto a las otras, en movimiento o reposo. El interés se centra entonces en las leyes del movimiento.

Si el universo es infinito (o àl menos ilimitado, como en algún momento propone Descartes siguiendo al Cusano), al ya no haber un centro del universo, se desvanece la distinción aristotélica entre movimiento natural y violento, y las mismas leyes regirán para todas y cada una de las partes de la materia.

La Primera es que toda parte de la materia continúa siempre existiendo en un mismo estado mientras el encuentro con otra no la obligue a cambiar. ${ }^{13}$

Esta ley es reformulada en los Principios II-37 como: “... cada cosa en particular persiste en el mismo estado tanto como sea posible y nunca lo cambia a menos que se produzca un encuentro con otra".

Si esta ley de inercia cartesiana pudiera ser sacada de contexto quizá no necesitaría de ningún fundamento ulterior, aunque sí de la demos-

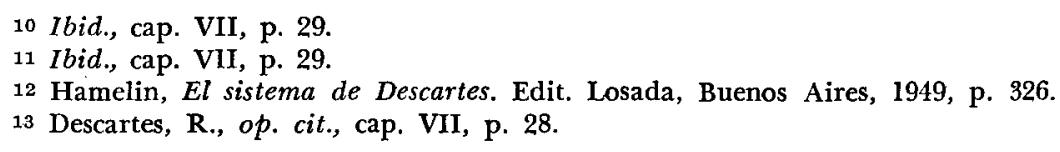


tración matemática; pero la formulación cartesiana no es autónoma - y con esto no me refiero al contexto histórico en el cual evidentemente la formulación de la ley en $E l$ Mundo antecede a la de Galileo y recoge, para la mayoría de los estudiosos, algunas propuestas de Kepler-, sino que me refiero a que la ley de inercia no es autómata de su concepción general del movimiento y la materia.

Habrá que leer: el mundo es un enorme conglomerado de partículas que ganan y pierden movimiento necesariamente por choques; así se enuncia la segunda ley de movimiento (Ley de permanencia, Ley de resistencia) en El Mundo:

Supongo como segunda regla que cuando un cuerpo empuja a otro no podría darle ningún movimiento si no perdiera al mismo tiempo el suyo, ni quitárselo sin que el suyo aumentara otro tanto. ${ }^{14}$

Para los fines de este trabajo lo que más importa no son los problemas que para la física se derivan de estas dos reglas, sino su fundamentación en la metafísica. En otras palabras, ¿es verdad, como lo afirman algunos estudiosos, que la física y por ende la cosmología cartesiana se fundan en la metafísica?

Con relación a este problema, dejando a un lado la consabida concepción del conocimiento humano que Descartes sostiene, en el Tratado se ofrece toda clase de testimonios que lo corroboran, desde los meramente enunciativos hasta las argumentaciones más sofisticadas.

A pesar de que para Descartes el movimiento no es una sustancia sino que en ocasiones lo refiere como modo o propiedad y en otras como relación, al considerar su causa lo "sustancializa" y encuentra que esta realidad no depende sino de Dios.

Si la cantidad de movimiento con que Dios dotó al mundo permanece inalterable, ello se debe a que Dios es inmutable y actúa siempre del mismo modo, lo cual constituye parte de su perfección. Así, la cantidad de movimiento generado en la creación permanece inalterable mientras Dios la conserva continuamente de instante a instante. Se hace evidente, por tanto, que lo que es realmente constante es el poder divino que actúa continuamente en el mundo, y por tanto la cantidad constante de movimiento deriva de la permanencia de Dios. ${ }^{15}$

En este sentido Brehier afirma que la ley de la permanencia de la cantidad de movimiento que descansa sobre la inmutabilidad de Dios es a

14 Ibid., cap. VII, p. 30.

15 Hoffding, Harold, $A$ history of Modern Philosophy. Dover Publications. Nueva York, 1955, p. 229. 
la física lo que la garantía divina de la evidencia es a la teoría del conocimiento. ${ }^{16}$

Pero dejemos hablar al propio Descartes quien en El Mundo afirma:

Así, estas dos reglas se siguen manifiestamente sólo de esto, que Dios es inmutable, y que actuando siempre del mismo modo produce siempre el mismo efecto. ${ }^{17}$

Si por medio de la primera ley sabemos que un cuerpo es incapaz de cambiar por sí mismo su estado de reposo o movimiento, en tanto que la causa última de todo movimiento es Dios ya que no existen potencias o virtudes intrínsecas en los cuerpos que causen su propio movimiento; si, por otro lado, es claro que la cantidad de movimiento permanece constante, como afirma la segunda ley, porque la inmutabilidad de Dios así lo garantiza y finalmente la única posibilidad para la transmisión del movimiento entre los cuerpos es el choque, parece consecuente afirmar que el choque es la única posibilidad de modificación de los cuerpos, lo cual significa que se trata de una acción instantánea. Así en el universo, tal y como se nos presenta en el Tratado, el movimiento se reduce a la acción instantánea por choques. El problema es cómo lograr la continuidad de un movimiento a través de sus múltiples acciones instantáneas. Este problema al menos intuitivamente parece ser paralelo al problema del pleno discreto pero contiguo. En efecto, la exclusión del vacio apelando a que las partes de la materia deben tocarse por todos lados hacen que el choque se transmita instantáneamente, permaneciendo constante la cantidad de movimiento, que se remite en última instancia a la inmutabilidad de Dios. De hecho las leyes del choque expresan todas la necesidad de que la cantidad del movimiento se conserve la misma antes y después del choque:

La tercera ley de movimiento se enuncia en El Mundo:

Agregaría en la tercera que mientras que un cuerpo se mueve, aunque su movimiento sea a menudo en línea curva y que no pueda jamás hacer ninguno que no sea en alguna forma circular, como quedó dicho más arriba, de cualquier modo cada una de sus partes individualmente tiende siempre a continuar el suyo en línea recta. $\mathrm{Y}$ así su acción, es decir, la inclinación que tiene a moverse, es diferente de su movimiento. 18

Esta regla también tiene como fundamento la inmutabilidad de Dios pero con una especificación interesante: la conservación continua.

10 Brehier, Histoire de la Philosophie. Presses Universitaires de France, Paris, 1960, p. 91 .

17 Descartes, R., op. cit., cap. VII, p. 32.

18 Ibid., cap. VII, p. 33. 
Esta regla se apoya en el mismo fundamento que las otras dos y no depende sino de que Dios conserve cada cosa por una acción continuada y en consecuencia no la conserva tal y como pudo haber sido en algún tiempo anterior sino precisamente tal y como es en el preciso instante en que la conserva. ${ }^{19}$

Aunque Descartes encuentra claro el fundamento de la tcrccra ley, lo cierto es que plantea algunas dificultades.

El movimiento que Dios transmite al universo es el movimiento uniforme y rectilíneo. Por otro lado, hay en todas las partes de la materia la tendencia a continuar este movimiento; sin embargo, la disposición, esto es, la distribución de las partes y las relaciones que en su ubicación se engendran, aunadas al hecho de que la materia constituye un pleno que aunque discreto es contiguo, dan como resultado que el movimiento registrado efectivamente en el mundo sea circular.

Descartes distingue entre el movimiento actual y la tendencia al movimiento. El movimiento actual es circular, curvo, etcétera, pero la tendencia auténtica de las partes de los cuerpos, consideradas individualmente, es siempre al movimiento rectilíneo. Así, el movimiento circular se explica más como efecto de fuerzas que se equilibran que como principio incito en las partes. Tal idea parece estar en estrecha relación con su concepción geométrica del espacio; en el espacio plano sólo se originan rectas en todos los sentidos imaginables pero siempre rectas; las curvas sólo aparecen como sumas de fuerzas, cabría decir desde la física, vectoriales y, desde la geometría analítica, como resultado de ecuaciones que se formulan tomando en consideración las variables de los dos ejes $x-y$.

Del planteamiento anterior se desprenden algunas consecuencias importantes:

1. El movimiento rectilíneo va a ser entendido por Descartes como movimiento enteramente simple, por tanto evidente y comprensible intuitivamente.

2. Cada partícula individual tiende a moverse en línea recta; para comprender el movimiento circular hay que concebir al menos dos partículas y la relación entre ellas.

3. En las partes en si mismas no hay nada que explique el movimiento circular; si se las considera aisladamente todo lo que ellas tienen es la tendencia a moverse en línea recta; el movimiento circular se da como una determinación externa.

4. El movimiento rectilíneo es una tendencia de las partes y es propiamente el movimiento que Dios creó, ya que es el autor del movimiento

19 Ibid., cap. VII, p. 33. 
en tanto movimiento uniforme y rectilíneo, pero son las diversas disposiciones de la materia las que lo tornan curvo. Dios, pues, es la causa del movimiento pero el efecto es a la vez múltiple y diversificado. Las partes retienen o transmiten el movimiento por múltiples choques, así el movimiento deja de ser uniforme y rectilíneo. Por otra parte las disposiciones de la materia permiten que sus partes sean fácilmente desviadas del movimiento rectilíneo. Tal situación nos lleva a concluir que sólo Dios en su infinita sabiduría podría dar cuenta de la diversificación y multiplicidad del efecto, porque ninguna física podría comprometerse a dar razón de cada caso del movimiento compuesto. ¿Cómo predecir el movimiento de una partícula si está sujeta a innumerables choques? El dilema cartesiano es sostener a la vez la ley de la conservación del movimiento y el movimiento compuesto. Descartes creyó encontrar la salida a este problema distinguiendo el movimiento de su determinación. ${ }^{20}$

El movimiento de las partes queda fundado en Dios mediatamente, pero la causa inmediata es la transmisión del movimiento por choques entre las partes de la materia; la pérdida y ganancia del movimiento debe especificarse a fin de distinguir entre el movimiento y su determinación, esto es, dirección y velocidad que habrán de seguir las partes. Esto no se especifica en el Tratado sino en los Principios. Sin embargo anticipa que:

Podría proponer aquí muchas reglas para determinar en particular cuándo, cómo y cuánto puede ser desviado el movimiento de cada cuerpo y aumentado y disminuido por su encuentro con otros, lo cual comprende sumariamente todos los efectos de la naturaleza. Pero me conformo con advertirles que además de las tres leyes que he explicado, no quiero suponer otras que aquellas que se siguen infaliblemente de estas verdades eternas, sobre las que los matemáticos están acostumbrados a apoyar sus más seguras y sus más evidentes demostraciones. ${ }^{21}$

De la explicación sobre la materia y el movimiento que en El Mundo propone Descartes puede concluirse que:

1. La cosmología está indisolublemente unida a la física desde la perspectiva no tanto del origen del mundo cuanto de la explicación del mundo actual.

2. Que a su vez la física no puede desvincularse de la metafísica, en

20 Hamelin, op. cit., Hamelin planteo el problema negativo. Descartes debió o bien renunciar a la ley de la conservación del movimiento o bien negar la composición del movimiento.

21 Descartes, R., op. cit., cap. VII, p. 35. 
primer lugar, porque pone en crisis los conceptos metafísicos tradicionales de materia y movimiento, esto es, en la polémica con los escolásticos muestra el paso en varios niveles del tratamiento metafísico al tratamiento físico de los problemas. Pero en segundo lugar, y mucho más importante, es que la física encuentra sus fundamentos últimos en la metafísica. En efecto, para la física cartesiana la postulación de la inmutabilidad de Dios, entendida como causa que produce siempre el mismo efecto, es fundamental, ya que sólo la regularidad inalterable de la relación causal puede garantizar la construcción de una ciencia física universal y necesaria.

3. El papel de Dios como creador del universo o, más específicamente, como creador de toda la materia que está a nuestro alrededor, concebida esencialmente como extensión ilimitada, es mucho menos relevante que el papel que la metafísica cartesiana asigna al concepto de Dios como causa última del movimiento. En efecto, si Dios es perfecto es inmutable $y$, ¿qué mejor fundamento o reforzamiento de la relación causal?

Descartes expresa al respecto: "Porque, ęcuál fundamento más firme y más sólido puede uno encontrar para establecer una verdad, incluso queriéndolo escoger a capricho, que el tomar la firmeza misma y la inmutabilidad que está en Dios?" 22

- Si Dios es inmutable garantiza la permanencia de la misma cantidad de movimiento (la. ley de movimiento).

- Si Dios es inmutable garantiza, al ser su acción idéntica, que conserva al mundo desde que lo creó tal y como es en cada instante (con la misma tendencia al movimiento uniforme y rectilíneo, pero con diversas disposiciones) en el sentido de que una vez dotada la materia del movimiento (ley de inercia), sus leyes (como leyes de la naturaleza) deben dar cuenta de todos los cambios que sufre.

Puesto que de esto sólo, a saber, que Dios continúa conservándola, se sigue necesariamente que debe de haber muchos cambios en sus partes, los cuales, me parece, no pueden ser atribuidos a la acción de Dios, puesto que ella no cambia nunca, por lo que los atribuyo a la naturaleza; y a las reglas mediante las cuales se producen estos cambios las llamo leyes de la naturaleza. ${ }^{23}$

- Si Dios es inmutable, ello garantiza que el instantaneísmo del movimiento por choques tenga continuidad en dos sentidos: $a$ ) en el sentido de la contigüidad de las partes de la materia que excluye el vacío y $b$ ) en 
el sentido de la transmisión inmediata encadenada del movimiento. No hay excepciones a la ley natural; Dios no opera milagros.

La conclusión inmediata que parece seguirse de lo antes dicho es que el supuesto fundamental de la física cartesiana, y por ende de la cosmología, es la inmutabilidad de Dios, entendida como causa que produce siempre el mismo efecto. En otras perspectivas, sin embargo, lo que Descartes requiere es la uniformidad o reforzamiento de la ley causal, lo cual le permita construir una ciencia física a cuya base se hallen los conceptos de uniformidad de la materia, ley de inercia y la transferencia del movimiento por choques.

Cabe entonces cuestionar qué es lo metodológicamente anterior: ¿La idea de Dios que parece dar sustento a las leyes de la naturaleza?, o ¿jon la concepción misma de la extensión y el movimiento las que determinan la necesidad de reforzar la relación causal para lo cual Descartes encuentra apropiado recurrir a la inmutabilidad divina?

En otros términos, la física cartesiana deriva verdaderamente de la postulación previa de la perfección e inmutabilidad de Dios o la concepción físico geométrica de la naturaleza (materia como extensión y mecanismo), que ha de expresarse en leyes; recurre a la postulación de Dios por parecerle a Descartes la vía más expedita de garantía de estabilidad, regularidad y constancia de los procesos naturales.

Esta segunda alternativa de explicación se ve fuertemente reforzada por comparación con un proceso metodológico similar que Descartes lleva a cabo en el ámbito de la epistemología, cuando funda la garantía de la existencia formal de las cosas, esto es, fuera del pensar, en la bondad divina.

Así, podemos concluir que los principios metafísicos (específicamente la inmutabilidad divina) no son en Descartes genéticamente punto de partida para la construcción de la física y la cosmología, sino que se postulan para sostener cierta concepción físico-cosmológica previamente asumida.

Continuamente oímos hablar de hipótesis ad hoc. En este caso tal vez podamos hablar de principios ad hoc cuya función parece dar solución a problemas que la teoría no puede resolver desde dentro. En Descartes, por ejemplo, el instantaneísmo de la acción mecánica, el movimiento en el pleno, la diversificación del efecto, a la par que la conservación de la misma cantidad de movimiento, son problemas que se originan o por la forma en que se han definido los términos y se han traducido en ley, o por cierta inconsistencia entre las propias leyes.

Aunado a lo anterior se da el supuesto no explícito de que es necesario que la relación causa-efecto se mantenga inalterable en todos los procesos de la naturaleza para poder formular sus leyes. 
Finalmente, a manera de conclusión muy general, parece seguirse de este análisis que el estudio sistemático de las ideas filosóficas impide la parcelación, y más bien invita a seguir el curso de la interacción de las diversas disciplinas en vista de la riqueza y singularidad de las teorías, razón por la cual al estudiar la cosmología cartesiana no hemos podido dejar a un lado ni la física ni la metafísica, ya que en cierta medida la constituyen desde dentro. La física aporta sus definiciones y leyes básicas a la cosmología, en tanto la metafísica le proporciona los principios de sustentación. 